(c) 1999 International Press

Adv. Theor. Math. Phys. 5 (1999) 1415-1443

\title{
On the Gauge
}

\section{Theory/Geometry \\ Correspondence}

Rajesh Gopakumar and Cumrun Vafa

Lyman Laboratory of Physics

gopakumr@tomonaga.harvard.edu

Harvard University

Cambridge, MA 02138

vafa@string.harvard.edu

\begin{abstract}
The 't Hooft expansion of $S U(N)$ Chern-Simons theory on $S^{3}$ is proposed to be exactly dual to the topological closed string theory on the $S^{2}$ blow up of the conifold geometry. The $B$ field on the $S^{2}$ has magnitude $N g_{s}=\lambda$, the 't Hooft coupling. We are able to make a number of checks, such as finding exact agreement at the level of the partition function computed on both sides for arbitrary $\lambda$ and to all orders in $1 / N$. Moreover,
\end{abstract}

e-print archive: http://xxx.lanl.gov/hep-th/9811131 
it seems possible to derive this correspondence from a linear sigma model description of the conifold. We propose a picture whereby a perturbative D-brane description, in terms of holes in the closed string worldsheet, arises automatically from the coexistence of two phases in the underlying $U(1)$ gauge theory. This approach holds promise for a derivation of the AdS/CFT correspondence.

\section{Introduction}

The large $N$ limit of gauge theories has been much studied since ' $t$ Hooft [30], in the hope of a string description. The essential idea is the following: when the 't Hooft coupling $\lambda=g_{Y M}^{2} N \ll 1$ we have an ordinary Feynman diagram description (with each diagram viewed as a Riemann surface with many holes). The proposal has been that, in the strong coupling limit $\lambda \gg 1$, there exists a closed string description (the holes being somehow filled in or condensed). A concrete and well studied example of this is large $N$ 2d Yang-Mills [17] where a quasi-topological closed string description [9] emerges at large $N$. Some tentative attempts were also made in a similar direction for large $N$ Chern-Simons theory [26], [10]. Recently, the large $N$ limits of certain non-trivial superconformal gauge theories have been concretely proposed to be closed superstrings on $A d S \times M$ backgrounds [21], [18], [36]. Extensions of the proposal in various directions as well as many non-trivial tests have been successfully carried out.

The aim of this paper is twofold: First, we propose a new duality the large $N$ limit of $S U(N)$ Chern-Simons theory on $S^{3}$ is exactly the same as an $N=2$ topological closed string on the $S^{2}$ blow up of the conifold geometry. Since both sides of the duality have been relatively well studied, we can compare, for instance, the partition functions on both sides. We find a strikingly exact match for all $\lambda$ (which plays the role of $1 / \alpha^{\prime}$ ) and to all orders in $1 / N$. (In [14], we had computed the closed string partition function for the $S^{2}$ blowup geometry of the conifold using M-theory, and had partially anticipated the connection to Chern-Simons theory which we fully develop here.) We are also able to successfully compare the coupling of the gauge theory to gravity, with quantities on the closed string side. This will also exhibit the by 
now familiar $U V / I R$ relation [28] . Though we have not made a detailed comparison, observables such as Wilson loops in the gauge theory (which compute knot invariants) should be given by holomorphic surfaces in the $S^{2}$ blown up geometry with a boundary which approaches a knot configuration on the $S^{3}$ at infinity.

This conjecture is very much in the spirit of the AdS/CFT correspondence in that the $S^{3}$ Chern-Simons theory has a topological open string description where we put $N$ (topological) 3-branes on an $S^{3}$ inside a conifold $T^{*} S^{3}$ background [33]. And the dual description we propose, is in terms of the same topological string, but now with no open strings, and on a modified background with parameters depending on $\lambda=g_{s} N$. In our case, the complexified area $t$ of the $S^{2}$ (i.e. with the B-field as its imaginary part) has the identification $t=i g_{s} N=i \lambda$. Intuitively, one can think of the $S^{2}$ as arising from the cotangent direction of $T^{*} S^{3}$ and corresponds to the two-sphere surrounding the positions of the 3 -branes in this transverse " $R$ ". One may also arrive at this correspondence, as we will explain in section 3, by considering the coupling of the Chern-Simons theory to gravity in the bulk. This will be seen to be quite similar to the way in which the conformal anomaly of the $N=4$ Super Yang-Mills manifests itself on the gravity side.

However, here we can actually do more: We suggest a simple derivation of this correspondence by considering a 2d QFT on the closed string side, which flows in the IR to the conformal field theory on the worldsheet. Examining this QFT in the limit $g_{s} N \propto t \rightarrow 0$, we find that there are two coexisting phases. In one phase ("Coulomb") the theory is free abelian in the IR and can be integrated out, leaving "holes" on the worldsheet. While in the other ("Higgs"), it is non-trivial and gives the bulk of the worldsheet theory. Moreover the worldsheet fields in the Higgs phase, satisfy Dirichlet conditions at the boundaries, which gives rise to an equivalent D-brane description in this limit.

This story parallels a similar one in the study of superstring vacua: The correspondence (duality) between Landau-Ginzburg/Calabi-Yau sigma models [16], [24] was explained in [35] by studying a one parameter family of $2 \mathrm{~d}$ QFTs with extra degrees of freedom in the UV. The resulting CFT's in the IR provide a smooth interpolation between the two descriptions. In fact, the 2d QFT (linear sigma model) description of the $S^{2}$ resolution of the conifold, which we use in this paper, was 
already proposed in [35]. This linear sigma model is just a $U(1)$ gauge theory with a single phase, the Higgs phase, when $t \neq 0$. As $t \rightarrow 0$ the Coulomb branch also opens up. Moreover, the topological string amplitudes, which are dominated by both non-linear sigma model instantons and $U(1)$ gauge instantons (vortex configurations), consist in this limit of only the latter. These vortices, which, in the generic Higgs phase, are point-like in the IR limit, can, in the $\lambda=0$ limit, have an arbitrary size. It is the "core" of these vortex lines that we will identify with the filled holes of worldsheet D-branes. We believe this procedure of replacing D-brane rules of worldsheet amplitudes in terms of a closed string theory description is bound to have other applications. ${ }^{1}$ In particular, it might be possible to prove the AdS/CFT conjectures along such lines.

The organization of this paper is as follows: In section 2 we state the large $N$ conjecture for $S U(N)$ Chern-Simons theory on $S^{3}$. In section 3 we perform the above mentioned explicit checks on this conjecture as well as provide further rationales for it. In section 4 we outline a derivation of this correspondence in terms of the 2-d linear sigma model. Finally in section 5 we make suggestions for some generalizations of the ideas presented here.

\section{The Conjecture}

Before going into the details of the particular conjecture which is the subject of this paper, it will be useful to set out some generalities regarding the open string description of large $N$ gauge theories and their conjectured relation to a closed string dual. This will also help in highlighting the similarities between our present conjecture and the AdS/CFT ones.

Take any large $N$ gauge theory containing only fields in the adjoint. The perturbative Feynman diagrams that contribute to the free energy

\footnotetext{
${ }^{1}$ The idea of condensing Dirichlet holes using extra degrees of freedom on the worldsheet, was already anticipated in [15] (as was pointed out to us by Albion Lawrence). Even though the precise description we find differs from theirs, (we have a two phase system which generates the surfaces with holes), there are some resemblances between the two approaches.
} 
(or more generally, gauge invariant correlation functions) admits the following 't Hooft organization:

$$
F=\sum_{g=0, h=1} C_{g, h} N^{h} \kappa^{2 g-2+h}=\sum_{g=0, h=1} C_{g, h} N^{2-2 g} \lambda^{2 g-2+h} .
$$

Here $\kappa$ is the ordinary gauge coupling $\left(g_{Y M}^{2}\right.$ for a Yang-Mills theory and $\frac{2 \pi}{k+N}$ in the Chern-Simons theory), while $\lambda=\kappa N$ is the 't Hooft coupling which is held fixed in the large $N$ limit. In writing Eqn. (1) , we have used the fact that a Feynman diagram in the double line notation can be thought of as a triangulation of a closed Riemann surface. Thus, in a diagram with $h$ faces, $V$ vertices and $E$ edges, we get the factor of $N^{h} \kappa^{E-V}$.

This also looks like an open string expansion on worldsheets with $g$ handles and $h$ boundaries. The double line notation is here seen as representing the world lines of the endpoints of the open string. In fact, in the case of Chern-Simons theory on a 3-dimensional manifold $M,{ }^{2}$ it was shown by Witten [33] that the coefficient $C_{g, h}=Z_{g, h}$, where $Z_{g, h}$ is the partition function of an open string theory on a worldsheet with $g$ handles and $h$ boundaries. The theory in this case is the A-model topological open string theory with $N$ "topological" D-branes on $M$ in an ambient six dimensional target space $T^{*} M$.

A similar thing happens in $N=4$ Super Yang-Mills in $d=4$. (Though the free energy vanishes here due to supersymmetry, correlation functions have a similar expansion. The $C_{g, h}$ 's will now carry momentum dependence as well.) This theory arises as the low energy limit of Type IIB open strings in flat space with a background of $N$ D-3 branes. And we can once again identify the genus $g$ and holes $h$ of the gauge theory diagram with those of the worldsheet.

In all these cases, one might ask as to what happens when one carries out the sum over the holes $h$ in Eqn. (1) (or the appropriate generalization). We expect something of the form

$$
F=\sum_{g=0} N^{2-2 g} F_{g}(\lambda)
$$

\footnotetext{
${ }^{2}$ Chern-Simons theory on $M$ is defined via the action $S_{C S}=\frac{k}{4 \pi} \int_{M} \operatorname{Tr}(A d A+$ $\frac{2}{3} A^{3}$ ). See [34] for further details.
} 
which is more like a closed string expansion. The question, since 't Hooft, has been: what is this closed string theory? ${ }^{3}$ Maldacena has proposed an answer in the case of $N=4 S U(N)$ Super Yang-Mills. It is closed IIB string theory on a background which is $A d S_{5} \times S^{5}$ with an $\mathrm{RR}$ field and a curvature scale set by $\lambda$ and with $g_{s} \propto \frac{\lambda}{N}$. Despite all the remarkable amount of evidence for it, we do not yet have a direct demonstration of this statement. We note the striking feature of this conjecture that by the time we have summed over all the holes the background has transmuted from $D 3$ branes in flat $R^{10}$, to the curved $A d S_{5} \times S^{5}$ without any branes. In both regimes, there exists a string theory description and this conjecture can be viewed as a statement about a 2d QFT on the worldsheet which interpolates between them.

Here, we will propose an answer for the case of $S U(N)$ ChernSimons theory on $S^{3}$. The claim is that it is exactly dual to the A-model topological closed string theory on the $S^{2}$ resolved conifold geometry. Let us first briefly review what this means.

The A-model topological closed string theory on a Calabi-Yau manifold $M$ is described by a "twisted" sigma model on $M$ [32]. This enormously simplifies the theory and the contribution to the string path integral comes solely from holomorphic maps $X(z)$ onto $M$. Besides the constant maps, these consist of 'instantons' which are topologically nontrivial mappings from the genus $g$ worldsheet onto two dimensional surfaces in $M$. We will write down the resulting structure of the partition function for genus $g$ in the next section, when we make a detailed comparison. The other feature of the topological A-model that we will need is that it is independent of the complex structure deformations of $M$ and depends only on the kahler ones. The particular Calabi-Yau $M$ that we are proposing in our dual is the local geometry near a conifold singularity which has been resolved by an $S^{2}$. We will be led to the identification of $\lambda$ with the B-field flux through the $S^{2}$ and $g_{s}=\frac{i \lambda}{N}$.

As we mentioned earlier, the $S U(N)$ Chern-Simons theory itself arises from the open string version of the topological A-model in the

\footnotetext{
${ }^{3}$ In carrying out this sum we have assumed the existence of a radius of convergence. This is by no means obvious. 't Hooft has established nice convergence properties for planar diagrams in a number of field theories [31] . And we will explicitly see this for all genus in the Chern-Simons theory. But even with a finite radius of convergence we could run up against the issue of a phase transition separating the perturbative and the stringy regime [11].
} 
presence of D-branes. In fact, the A-model open string theory on the Calabi-Yau $T^{*} S^{3}$ with Dirichlet boundary conditions on the $S^{3}$ gives rise to the $S U(N)$ gauge theory on $S^{3}$ in the manner outlined below Eqn. (1). The $T^{*} S^{3}$ geometry happens to be the other side of the conifold. In other words when the singularity has been resolved by an $S^{3}$. So we see that, just as in the AdS/CFT cases, summing over holes has made the original $T^{*} S^{3}$ undergo the conifold transition to the resolved geometry with no branes. (See Fig. 1.)
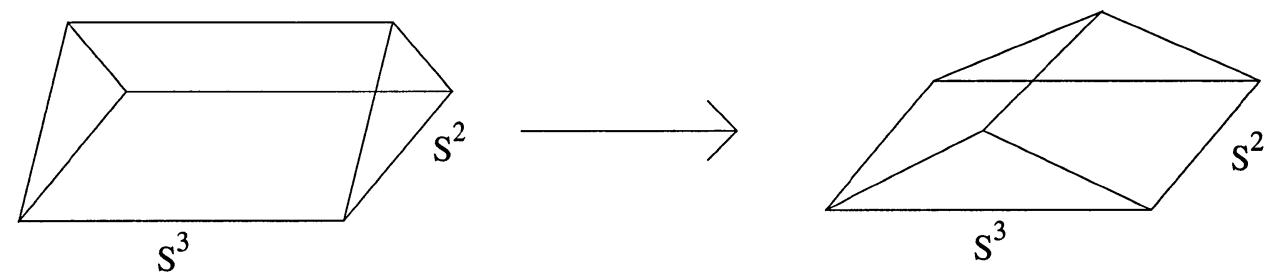

Figure 1: The geometry of $T^{*} S^{3}$ with an $S^{3}$ of finite size goes into an $S^{2}$ resolved geometry after the conifold transition.

As the figure indicates, the geometry of the conifold is essentially like a cone with a base which is topologically $S^{2} \times S^{3}$. In the $S^{2}$ resolved geometry on the right, the space at infinity is $S^{2} \times S^{3}$ with the $S^{2}$ of finite size. This is like the analogous $S^{5}$ of the Maldacena conjecture. The gauge theory itself can be thought of as living on the large $S^{3}$. In fact one can push the analogy even further: In the $A d S^{5} \times S^{5}$ description of $N=4$ Yang-Mills, (in Euclidean version) the boundary is $S^{4} \times S^{5}$ with the radial direction of $A d S_{5}$ filling in the $S^{4}$. In the weak coupling regime, the boundary is still $S^{4} \times S^{5}$ but the difference is that now it is the $S^{5}$ that gets filled. So in some sense there is already a conifold like transition in the $N=4$ Yang-Mills as well, when we go from weak to strong coupling. In fact, Fig. 1 for the conifold is topologically also accurate for this case if we replace $S^{3} \rightarrow S^{4}$ and $S^{2} \rightarrow S^{5}$.

More detailed aspects of the conifold geometry and its blow up will be discussed in section 4 . Here we would like to remark that, in a certain geometrical sense we are exactly at the transition point. On the gauge theory side the amplitudes do not depend on the size of the $S^{3}$ (as it is topological) and so we can take it to have shrunk to zero size. On the closed string side, the actual area of the $S^{2}$ is zero - it is only the $B$-field which is turned on. This will prove important in deriving the $\mathrm{D}$-brane rules from the closed string theory description, as 
we will see in section 4 .

Let us remark in passing that this conjecture provides an example of a closed string dual of a non-supersymmetric gauge theory that lives in three (rather than one) higher dimensions.

\subsection{Wilson Loop Observables}

Next we turn our attention to Wilson loops in the Chern-Simons theory which are the celebrated knot polynomials [34]. Consider a loop $\Gamma$ in $S^{3}$ and the Wilson loop observable of the Chern-Simons theory $\langle W(\Gamma)\rangle=$ $\left\langle\operatorname{Tr} P \exp \left(i \int_{\Gamma} A\right)\right\rangle$ in the fundamental representation of $S U(N)$. We can generalize this by considering arbitrary representations as well as an arbitrary number of Wilson loops. For simplicity let us restrict our attention to a single loop in the fundamental representation. We would like to give a prescription for computing it on the closed string side. In order to do this, we will need some facts about the topological A-model on worldsheets with boundary, which we will now review.

It is known that the boundary should be mapped onto a Lagrangian 3-dimensional submanifold $C_{3} \subset M$ of the Calabi-Yau 3-fold [33]. Being Lagrangian means that the Kahler form $k$ vanishes when restricted to $C_{3}$. As we said earlier, the path-integral in the A-model is dominated by holomorphic Riemann surfaces. Then the condition that these end on a Lagrangian submanifold implies that the neighborhood near the boundary is mapped to a half cylinder which is orthogonal to $C_{3}$.

Let $f$ be an isolated holomorphic map from the worldsheet $\Sigma$ to the Calabi-Yau $M$ with one boundary on $C_{3}$. The fact that it is holomorphic implies that the area is given by

$$
A(\Sigma)=\int_{\Sigma} f^{*}(k)
$$

where $f^{*}(k)$ denotes the pull back of $k$ on the worldsheet. Such a map will have a contribution of $\pm \exp (-A)$ to the partition function (the \pm is determined by certain ratio of determinants [33] ). Now, given another holomorphic map $f^{\prime}$, with a similar $\pm \exp \left(-A^{\prime}\right)$ contribution, let us look at the difference $A^{\prime}-A$. Let $\gamma$ and $\gamma^{\prime}$ denote the boundaries of $\Sigma$ and $\Sigma^{\prime}$ in $C_{3}$ respectively. Consider a 2-dimensional submanifold 
$B_{2} \subset C_{3}$, whose boundary is $\partial B_{2}=\gamma-\gamma^{\prime}$ and the closed 2-surface $\hat{\Sigma}$, in $M ; \hat{\Sigma} \equiv f(\Sigma)+B_{2}+f\left(\Sigma^{\prime}\right)$. It is easy to see that with opposite orientations on $f(\Sigma)$ and $f\left(\Sigma^{\prime}\right)$, the boundary of $\hat{\Sigma}$ is empty. Let us consider the integral of $k$ on $\hat{\Sigma}$ :

$$
\int_{\hat{\Sigma}} k=-A+\int_{B_{2}} k+A^{\prime}=A^{\prime}-A
$$

where we have used the fact that $B_{2} \in C_{3}$ and that $k$ vanishes on $C_{3}$. Thus, we learn that the difference $A^{\prime}-A$ is given by the integral of $k$ on a closed 2-cycle $\hat{\Sigma}$ in $M$, which is thus quantized. In particular, if we have only one Kahler class, parameterized by the Kahler parameter $t$, then

$$
A^{\prime}-A=n t
$$

for some integer $n$.

With these preliminaries we are now ready to define a prescription for the computation of $W(\Gamma)$ in the $S^{2}$ blown up geometry. As in [22] it is natural to consider worldsheets whose boundary is a loop $\Gamma$ living on the boundary of the space. In our case the boundary is topologically $S^{2} \times S^{3}$. Fix a large $S^{3}$ of size $\Lambda$ on the boundary, which we take to be a Lagrangian submanifold. We consider holomorphic maps from the worldsheet with boundary and require the boundary to lie in $S_{\Lambda}^{3}$, and that the boundary intersect $S_{\Lambda}^{3}$ on the knot $\Gamma$ (or on a knot isotopic to $\Gamma)^{4}$. This is our proposal for computing Wilson loop observables on the closed string side.

Note that the area $A$ of surfaces ending on $S_{\Lambda}^{3}$ will depend on $\Lambda$. Moreover, as $\Lambda \rightarrow \infty$ this area will diverge. However the difference between two surfaces ending on $S^{3}$ will be independent of $\Lambda$ and finite, as explained above. Thus the proposed topological A-model observables are well defined up to an overall multiplication by $\exp \left(-A_{0}(\Lambda)\right)$. This is in fact to be expected, since on the Chern-Simons side, the Wilson loop observables are divergent due to UV singularities. They can be regularized by a choice of point splitting, known as 'framing' the knot

\footnotetext{
${ }^{4}$ Actually, the condition that the boundary be mapped to a fixed curve $\Gamma$ is too strong [29] and no such holomorphic map would generically exist. It seems more natural to fix the knot type $\Gamma$ in $S_{\Lambda}^{3}$. And then require that the boundary of the worldsheet be any loop $\Gamma^{\prime}$ which is smoothly deformable to $\Gamma$ (i.e. $\Gamma^{\prime}$ and $\Gamma$ are equivalent knots)
} 
[34]. Different framings alter $W(\Gamma)$ by multiplicative factors which we are now identifying with the choice in the overall area subtraction. In which case, remembering the quantization of area differences, we have the proposal

$$
\langle W(\Gamma)\rangle=\exp \left(-A_{0}\right) \sum_{\partial f_{n}\left(\Sigma_{g}\right) \sim \Gamma} \pm\left(g_{s}\right)^{2 g-1} \exp (-n t)
$$

where $f_{n}$ are holomorphic maps from a surface $\Sigma_{g}$ of genus $g$ with one boundary on $\Gamma$. If the holomorphic curves come in families one is expected to compute certain integrals over the moduli space of such curves, and the sum above should be understood in that sense. In the next section we will see some evidence that knot invariants on $S^{3}$ do indeed have this structure.

\section{Checks of the Conjecture}

The most striking check of this conjecture is the matching of the free energies. In other words, the genus $g$ contribution in 2 of the ChernSimons theory, will precisely match the genus $g$ partition function of the closed string theory on the $S^{2}$ resolved geometry. Next, we consider the anomalous gravitational coupling of the Chern-Simons theory and relate it to a similar "anomaly" on the closed string side. Finally, we also consider Wilson loop observables on the gravity side. In this case, since the configuration of holomorphic maps of interest have not been previously studied, we cannot check the statements in detail. We will, however, demonstrate that the structure of the knot invariants agrees with the general structure expected from the computations on the closed string side. Moreover, having gained confidence in our conjecture, we can instead turn this around and propose a reformulation of knot invariants in terms of a summation over minimal surfaces bounding the knot.

\subsection{The Free Energy}

The exact free energy of the $S U(N)$ Chern-Simons theory on $S^{3}$ [26] can be expanded in an 't Hooft expansion in a fairly straightforward 
way. The main point to be remembered is that the bare 't Hooft coupling $\lambda_{b}=\frac{2 \pi N}{k}$ is finitely renormalized to $\lambda=\frac{2 \pi N}{k+N}$ (as follows from the renormalization of $k$ [34]). The free energy contribution to $N^{2-2 g}$, written in the open string expansion of Eqn. (1), is (see appendix of [14] for details; here we will define our free energy via $Z=e^{-F}$, differing in overall sign from [14])

$$
\begin{aligned}
& F_{g}(\lambda)= \\
& -\chi_{g}\left[1+2 \sum_{p=1}^{\infty} \zeta(2 g-2+2 p)\left(\begin{array}{c}
2 g-3+2 p \\
2 p
\end{array}\right)\left(\frac{\lambda}{2 \pi}\right)^{2 g-2+2 p}\right]
\end{aligned}
$$

for $g>1^{5}$. Therefore the coefficients $C_{g, h}=Z_{g, h}$ in 1 have the simple form

$$
C_{g, 2 p}=-\chi_{g, 2 p} \frac{2 \zeta(2 g-2+2 p)}{(2 \pi)^{2 g-2+2 p}} ; \quad C_{g, 2 p+1}=0
$$

Here

$$
\begin{aligned}
\chi_{g, h} & =(-1)^{h}\left(\begin{array}{c}
2 g-3+h \\
h
\end{array}\right) \\
\chi_{g} & =(-1)^{h}\left(\begin{array}{c}
2 g-3+h \\
h
\end{array}\right)(-1)^{g-1} \frac{B_{g}}{2 g(2 g-2)}
\end{aligned}
$$

is the Euler characteristic of the moduli space of Riemann surfaces with genus $g$ and $h$ punctures.

The similar answer for genus zero and one (the coefficients of $N^{2}$ and $N^{0}$ respectively) takes the form

$$
\begin{aligned}
& F_{0}(\lambda)=\frac{3}{4}-\frac{1}{2} \ln \lambda-\sum_{p=2}^{\infty} \frac{\zeta(2 p-2)}{p-1} \frac{\left(\frac{\lambda}{2 \pi}\right)^{2 p-2}}{2 p(2 p-1)} \\
& F_{1}(\lambda)=-\sum_{p=1}^{\infty} B_{1} \frac{\zeta(2 p)}{2 p}\left(\frac{\lambda}{2 \pi}\right)^{2 p} .
\end{aligned}
$$

The sum over the number of holes $h=2 p$ can be carried out in all these cases.

${ }^{5}$ Note that the exact Chern-Simons expression has a $\lambda$ independent term. This and the $\ln \lambda$ terms in $F_{0,1}$ are present in the appendix of [14] but were not interpreted there. 
Genus zero: The sum in Eqn. (7) can be carried out (after taking two derivatives and rewriting $\left.\zeta(2 m)=\sum_{n=1} \frac{1}{n^{2 m}}\right)$.

$$
\begin{gathered}
N^{2} F_{0}(\lambda)=-\left(\frac{N}{\lambda}\right)^{2}\left[-\zeta(3)+i \frac{\pi^{2}}{6} \lambda-i\left(m+\frac{1}{4}\right) \pi \lambda^{2}+\right. \\
\left.+\frac{i \lambda^{3}}{12}+\sum_{n=1}^{\infty} \frac{e^{-i n \lambda}}{n^{3}}\right]
\end{gathered}
$$

(the integer $m$ in the above expression is not uniquely fixed-this is also echoed on the closed string side as we will note below). With the identifications, $g_{s}=\frac{i \lambda}{N}$ (the $i$ comes from the $i$ in the Chern-Simons action) and $t=i \lambda$ for the complexified Kahler parameter for the $S^{2}$, our answer reads as

$$
\mathcal{F}_{0}=\frac{1}{g_{s}^{2}}\left[-\zeta(3)+\frac{\pi^{2}}{6} t+i\left(m+\frac{1}{4}\right) \pi t^{2}-\frac{t^{3}}{12}+\sum_{n} \frac{e^{-n t}}{n^{3}}\right] .
$$

Let us compare this with a general genus 0 topological string amplitude for a Calabi-Yau with one Kahler class [8]

$$
\mathcal{F}_{0}=\frac{1}{g_{s}^{2}}\left[-\frac{\chi}{2} \zeta(3)-\frac{\pi^{2}}{6} c_{2} t+i \pi a t^{2}-C \frac{t^{3}}{3 !}+\sum_{n, m} d_{m} \frac{1}{n^{3}} \exp (-n m t)\right]
$$

Here $\chi$ is the Euler characteristic of the manifold ${ }^{6}, c_{2}$ the second Chern class (more precisely, it is $c_{2} t=\int k \wedge c_{2}$ that appears here [19]) and $C$ is the classical self-intersection number for the Kahler class (i.e. $C t^{3}=\int_{M} k \wedge k \wedge k$ ). Also $d_{m}$ denotes the number of primitive degree $m$ holomorphic spheres, and $n$ labels the multi-covering of the basic ones. The quantity $a$ in the above formula does not have a known topological interpretation; However for the case of Calabi-Yau with one modulus, it is predicted [19] that $a=C / 2 \bmod \mathbf{Z}^{7}$

\footnotetext{
${ }^{6}$ The coefficient $\zeta(3) \frac{\chi}{2}$ has its origin in the $R^{4}$ term of type II strings.

${ }^{7}$ From the viewpoint of closed string theory, only the third derivative and higher of $\mathcal{F}_{0}$ (and first and higher of $\mathcal{F}_{1}$ ) refer to physical quantities. However, mirror symmetry naturally dictates the above form [19] .
} 
The various terms in our expression 10 are now easily recognized! Let us first recall that in the $S^{2}$ resolved geometry there is only one primitive holomorphic sphere and it is of degree one, so that $d_{1}=1$ and $d_{i}=0$ for $i>0$. Moreover, we are led to the identifications:

$$
C=\frac{1}{2}, \quad c_{2}=-1, \quad \chi=2 .
$$

To compare these quantities with those in our $S^{2}$ blown up geometry, we have to recall that, for non-compact manifolds, some of these quantities are naively divergent and have to be carefully regularized. (This point will be crucial to the discussion in the next subsection). But we can nevertheless see why these assignments are natural for our geometry. The formal continuation of $t \rightarrow-t$ corresponds to a "flop" of the blow up geometry, under which the self-intersection number, of the $S^{2}$, undergoes a down shift by 1 [35], [2]. By requiring that the above expansion be valid on both sides of the flop, we fix $C=1 / 2$ (note that $t^{3}$ is odd under $t \rightarrow-t$ ). A similar argument shows [20] that under the flop $\int c_{2} \wedge k$ goes up by $2 t$ which fixes $\int k \wedge c_{2}=-t$ as a valid formula on both sides of the flop. The value of $\chi=2$ is also natural: $\chi$ is twice the difference between the number of Kahler and complex deformations. In the case at hand, we have only one of the former and none of the latter, giving $\chi=2$. Note that $\chi$ does not change under a flop, as expected.

Genus one: The genus one answer, 8 can also be easily written in closed form as

$$
N^{0} F_{1}(\lambda)=i \frac{B_{1}}{4} \lambda+\frac{B_{1}}{2} \ln \left(1-e^{-i \lambda}\right)
$$

Once again, with the above identifications we get

$$
\mathcal{F}_{1}=\frac{1}{24} t+\frac{1}{12} \ln \left(1-e^{-t}\right) .
$$

Again, we should compare this with the genus one answer of [5] (specialized to the case that there are no $g>0$ holomorphic curves)

$$
\mathcal{F}_{1}=-\frac{c_{2}}{24} t+\frac{1}{12} \sum_{m} d_{m} \ln \left(1-e^{-m t}\right)
$$


In our case, with the $d_{i}=\delta_{i 1}$ and $c_{2}=-1$ as above, 14 reduces precisely to 13 .

Genus $g>1$ : And finally, the non-trivial structure of higher genus terms (the sum over $p$ in 4 is carried out in [14]) is captured in

$$
\begin{aligned}
N^{2-2 g} F_{g}(\lambda)= & \left(\frac{N}{\lambda}\right)^{2-2 g}(-1)^{g-1} \times \\
& \times\left[(-1)^{g-1} \chi_{g} \frac{2 \zeta(2 g-2)}{(2 \pi)^{2 g-2}}-\chi_{g} \sum_{n \in \mathbf{Z}} \frac{1}{(\lambda+2 \pi n)^{2 g-2}}\right]
\end{aligned}
$$

which after rewriting the sum and translating into closed string variables reads as

$$
\mathcal{F}_{g}=g_{s}^{2 g-2}\left[(-1)^{g-1} \chi_{g} \frac{2 \zeta(2 g-2)}{(2 \pi)^{2 g-2}}-\frac{\chi_{g}}{2 g-3) !} \sum_{n=1}^{\infty} n^{2 g-3} e^{-n t}\right] .
$$

The structure of the closed topological string anticipated in this case is [6] :

$$
\mathcal{F}_{g}=g_{s}^{2 g-2}\left[\frac{\chi}{2} \int_{\mathcal{M}_{g}} c_{g-1}^{3}+\sum \alpha_{n} e^{-n t}\right]
$$

The first term in brackets is the contribution from constant maps - the whole worldsheet is mapped to a point. Here $c_{g-1}^{3}$ is a certain characteristic class on the moduli space of genus $g$ Riemann surfaces. The second term corresponds to the contribution of genus $g$ curves covering the sphere $n$ times and $\alpha_{n}$ are some universal coefficients. The contribution $\int_{\mathcal{M}_{g}} c_{g-1}^{3}$ from constant maps was recently obtained using the M-theory/ type IIA correspondence [14] and by using type IIA/heterotic duality [23] to be exactly given by

$$
\int_{\mathcal{M}_{g}} c_{g-1}^{3}=(-1)^{g-1} \chi_{g} \frac{2 \zeta(2 g-2)}{(2 \pi)^{2 g-2}} .
$$

With the $\chi=2$ in our case, we see that the first term in (17) matches with the first term in (16). This term has a simple physical interpretation [14]. It is seen as coming from a one loop integrating out (a la 
Schwinger) of charged zero branes in the physical IIA theory on the Calabi-Yau. The integral $\int_{\mathcal{M}_{g}} c_{g-1}^{3}$ was also derived via a direct computation by mathematicians [12]. The coefficients $\alpha_{n}$, corresponding to the contribution from multiply wound higher genus curves over $S^{2}$, were also derived using the Schwinger one-loop computation [14]. The final answer exactly matches that predicted in (16) (compare with Eqn. (3.3) in [14]). In fact, the form of the second term presented in (15) is that obtained by integrating out bound states of $S^{2}$ wrapped two branes and zero branes in the physical IIA theory. The $\alpha_{n}$ have again been computed directly by mathematicians [12] and the result agrees with that obtained from the Schwinger computation. Thus once again the large $N$ free energy agrees fully with the topological string partition function on the $S^{2}$ blow up!

\subsection{The Coupling to Gravity}

While the above detailed agreement of the free energies should convince even hard-boiled skeptics, we can actually match a few more quantities. In fact we can ask as to how the topological branes couple to gravity, which is after all the reason why the geometry is altered.

Formally one expects that the Chern-Simons theory is independent of the background metric. Indeed, the classical Lagrangian is background independent. However, it was shown in [34] that there can be a coupling at the quantum level via the gravitational Chern-Simons term. It was argued that, in a particular regularization one would need to add the gravitational Chern-Simons term as a counterterm in order for the Chern-Simons partition function to be purely topological. In other words, the free energy of Chern-Simons theory $F_{C S}$ is

$$
F_{C S}=F_{T o p}+i \pi \frac{c}{12} I(\omega)
$$

where

$$
I(\omega)=\frac{1}{8 \pi^{2}} \int_{S^{3}} \operatorname{Tr}\left(\omega d \omega+\frac{2}{3} \omega^{3}\right) .
$$

is the gravitational Chern-Simons action in terms of $\omega$ which is the spin connection and $c$ is the central charge of the $S U(N)$ WZW CFT 
at level $k$,

$$
c=\frac{k\left(N^{2}-1\right)}{k+N}=-\frac{1}{2 \pi}\left(N^{2}-1\right)(\lambda-2 \pi) .
$$

This result was motivated in [34] from a one-loop calculation. It has also been confirmed at the two loop level [3]. Furthermore, it was conjectured in [33] that this should be the only coupling between the closed string sector of the topological A-model with the open string sector that gives rise to the Chern-Simons theory.

The divergences in perturbative gauge theory which give rise to the gravitational coupling come from the ultraviolet. In the spirit of AdS/CFT correspondence one expects this to show up as an IR effect on the closed string side [28] . Indeed, there are such potential IR divergences. Consider, for example, topological A-model at genus one. It was shown in [5] that the leading term in the large area limit involves a term

$$
\mathcal{F}_{1}=\frac{1}{24}\left[\frac{1}{8 \pi^{2}} \int k \wedge \operatorname{tr}(R \wedge R)\right]+\ldots
$$

For compact manifolds this is a topological invariant in the form of $\frac{1}{24} \int k \wedge c_{2}$ where $c_{2}$ is the second Chern class of the manifold. However, for manifolds with boundary this is not a topological invariant. This is similar to the better known case of $\int R$ on 2-manifolds with boundaries, where it is no longer a topological invariant unless we add a boundary term. In the case at hand, the boundary is topologically $S^{3} \times S^{2}$ and it is the quantity

$$
\int k \wedge c_{2} \rightarrow \frac{1}{8 \pi^{2}} \int k \wedge \operatorname{tr}(R \wedge R)-\int_{S^{3}} I(\omega) \int_{S^{2}} k
$$

which is topological, where the $S^{2} \times S^{3}$ is identified with the boundary of the blow up geometry of the conifold. To see the topological invariance of this quantity, one uses the relation $\operatorname{Tr}(R \wedge R)=d L_{C S}(\omega)$. This implies that the topological closed string theory also has an "anomaly" on non-compact manifolds - one obtains a surface term which depends on the boundary metric.

$$
\mathcal{F}_{1}=\mathcal{F}_{1}^{\text {Top }}+\frac{1}{24} I(\omega) \int_{S^{2}} k
$$


This matches the structure we found in the Chern-Simons theory Eqn (18). Note that the dependence of the coefficient in (18), (19) predicts a genus 0 (the $N^{2}$ term) as well as a genus 1 contribution ( $N^{0}$ term). Let us first consider the genus 1 contribution and compare it with what we are finding from the topological string theory at genus 1 . To match the coefficient, we first need to shift $\lambda \rightarrow \lambda+2 \pi$ in Eqn (19). ${ }^{8}$ We then see that the coefficient of $N^{0} I(\omega)$ is $i \frac{\lambda}{24}$ matching that in (21), with the identification $t \equiv \int_{S^{2}} k=i \lambda$. Thus, the gravitational coupling has given us an independent derivation of this identification, or depending on your view, another check. Note that it is crucial that the group is $S U(N)$ rather than $U(N)$ for this match to work. This is also similar to what has been found in the AdS/CFT correspondences [36].

But we also have a genus zero contribution in Eqn. (19), i.e.

$$
\delta_{\text {grav }} F_{0}=-\frac{i}{24 g_{s}^{2}}(\lambda+2 \pi)^{2} \lambda I(\omega)
$$

where we have kept in mind the shift in $\lambda$ and that $\left(\frac{N}{\lambda}\right)^{2}=\frac{1}{g_{s}^{2}}$. We firstly note that there is no constant term, signifying that we do not get an $I(\omega)$ contribution from regularizing the Euler character term in (11). But we do have a linear term like in genus one which comes from the corresponding linear term in (11). Noting the substitution in (20), we see that the closed string again has the anomalous contribution

$$
\delta \mathcal{F}_{0}=-\frac{1}{g_{s}^{2}} \frac{\pi^{2}}{6} t I(\omega)
$$

This is again exactly what we see on the Chern-Simons theory side in the linear term of Eqn. (22). The quadratic term was anyhow ambiguous and did not seem to have a topological meaning. The cubic term in (22) is presumably seen on the closed string side after regularizing the topological $C=\int k \wedge k \wedge k$ term. It would be interesting to verify this.

To summarize, we find that the gravity counterterms in the gauge theory are reflected in similar non-topological infrared contributions

\footnotetext{
${ }^{8}$ This is the shift of the B-field by $2 \pi$. The instanton terms in brackets in (12) and (9) are obviously invariant under this shift. It is easy to check that the topologically meaningful coefficients of the constant, the linear and the cubic terms in (9) are also left unchanged. The only changes are in the anyway ambiguous quadratic and constant terms in $\mathcal{F}_{0}$ and $\mathcal{F}_{1}$ respectively.
} 
on the closed string side. The anomalous coupling to gravity in ChernSimons theory may be thought of as a simple analogue of the conformal anomaly in a conformal field theory which, again, arises from the regularization of UV divergences. In the AdS/CFT correspondence, the conformal anomaly in $N=4 \mathrm{SYM}$ was seen on the AdS side as an infrared effect coming from the necessity of truncating the $A d S_{5}$ to finite size and choosing a particular conformal structure [36], [27]. This very closely parallels our discussion above in the topological framework. We note that the central charge which measures the conformal anomaly in these cases also had only genus zero and one contributions (of which only the genus zero or supergravity contribution has been verified).

\subsection{Wilson Loops}

Being a topological gauge theory, the local observables of Chern-Simons theory are trivial. The absence of local degrees of freedom in the gauge theory is nicely matched with the absence of a physical graviton in the dual closed string theory. ${ }^{9}$ Instead, one can consider Wilson loop observables in the Chern-Simons theory. In the previous section, we made the conjecture that these are evaluated on the closed string theory side by summing over holomorphic maps ending on any loop, in the boundary $S^{3}$, which is isotopic to the given loop. If this is true, then there is a non-trivial zero'th order check: if we take the most general knot invariant on the Chern-Simons side and expand it in the large $N$ limit, does it admit being written in the form 3 , that we expect on the closed string side? This is what we will verify here, leaving the more detailed checks for later. Indeed, $\langle W(\Gamma)\rangle$ for $S U(N)$ give the knot invariants [34] studied by mathematicians. More precisely, it is the HOMFLY polynomial [1] which is traditionally written in terms of 2 variables, $P_{\Gamma}(q, z)$ with $q=e^{\frac{i \pi N}{k+N}}$ and $z=2 i \sin \left(\frac{\pi}{k+N}\right)$. It is interesting that when expressed in terms of closed string variables, these precisely parametrize the two independent quantities

$$
q=\exp \left(\frac{t}{2}\right), \quad z=2 i \operatorname{Sin}\left(g_{s} / 2\right)
$$

For instance, for the simplest loop $C$ which is topologically trivial

\footnotetext{
${ }^{9}$ However one can write down a topological gravity theory corresponding to it, known as "Kahler Gravity" [7].
} 
- not knotted at all, the answer is

$$
\begin{aligned}
\langle W(C)\rangle & =\frac{\left(q-q^{-1}\right)}{z}=\frac{e^{\frac{t}{2}}-e^{-\frac{t}{2}}}{2 i \sin \frac{g_{s}}{2}} \\
& =e^{\frac{(t-i \pi)}{2}}\left(1-e^{-t}\right)\left(\frac{1}{g_{s}}+\sum_{k=0} c_{k} g_{s}^{2 k+1}\right) .
\end{aligned}
$$

where the $c_{k}$ 's are some coefficients involving the Bernoulli numbers. This certainly looks like the instanton sum in 3, with the leading term coming from the disc (the $\frac{1}{g_{s}}$ term) and higher order terms being the analogue of the degenerate higher genus contributions that we saw in the partition function. We also see that there are only contributions with $n=0,1$ coming with opposite signs. The overall factor can be thought of as the framing ambiguity mentioned in Sec.3 . It would be interesting to understand all these features more precisely. ${ }^{10}$

For a general knot, the answer on the gauge theory side follows from the skein relations shown in figure 2 .

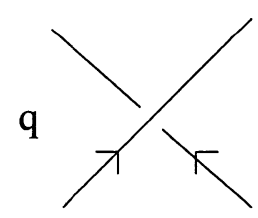

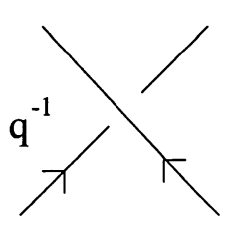

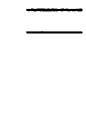

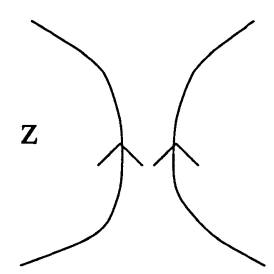

Figure 2: The skein relations.

The figure depicts manipulations on knots that can be performed at every crossing. The skein relations should be viewed as the ChernSimons analog of the loop equations studied in connection with Wilson loops. These relations can be used inductively to evaluate $\langle W(\Gamma)\rangle$ in terms of the simple loop average above. We see that we get extra factors of $z$ (or $g_{s}$ ) when we increase the number of disjoint components by the operation on the right hand side. Therefore, the leading term in $W(\Gamma)$

\footnotetext{
${ }^{10}$ Already the answer for the trivial knot given above seems suggestive: If we can deform the knot at infinity to a circle over $S^{2}$, the two contributions at the level of the disc should correspond to which way one wraps around $S^{2}$ as one bounds the circle. Moreover a disc with handles attached would need to have moduli, in order to reproduce the Sin contribution in the denominator. In fact this is very much reminiscent of the Schwinger-like contribution which gives the contribution of maps from higher genus to sphere, in that it is roughly speaking, a square root of it.
} 
for a single component loop will always be $O\left(\frac{1}{g_{s}}\right)$, as expected from a disc. The expansion will also have an odd parity with respect to $z$, i.e. with only odd powers of $g_{s}$. Finally, using the skein relation and (23) it is possible to convince oneself that only even powers of $q$ appear in $W(\Gamma)$ for a single loop (upto an overall $q^{\alpha}$ ). Noting that $q=e^{\frac{t}{2}}$, this has exactly the structure of (3) upto the overall framing ambiguity.

\section{The Linear Sigma Model and Deriva- tion of the Large N/Gravity Correspon- dence}

As we reviewed in Sec. 3, for gauge theories arising from D-branes, there are two limits which are relatively simple. One is the limit when $\lambda=g_{s} N \rightarrow 0$ and the other when $\lambda \gg 0$. Both limits are conjectured to be described by the same underlying string theory with the same small $g_{s}$, but with a modified geometry. Thus, this is not really an S-duality. Rather, it is closer in spirit to the different worldsheet descriptions that appear in different regions of target space parameters, as in CalabiYau/Landau-Ginzburg duality. And one would hope to be able to prove this duality along similar lines. Having said that, we should point out an important difference. A closed string sigma model description when $\lambda \gg 0$ is somehow going over to an open string one by the time $\lambda \rightarrow 0$. In other words, holes develop on the originally closed string world sheet. Is there an underlying dynamical principle that can give rise to this? We will now propose a closed worldsheet description, in terms of a linear sigma model, for all $\lambda$, including the limit $\lambda \rightarrow 0$. What will be special about the $\lambda \rightarrow 0$ limit is that, the worldsheet configurations in the IR will consist of two phases in coexistence. In one region the $2 \mathrm{~d}$ theory becomes trivial in the infrared and can be integrated out, whereas in the other, it corresponds to the sigma model on $T^{*} S^{3}$ with the $S^{3}$ shrunk to a point. Moreover, the fields on the boundary between these two regions will take values on the $S^{3}$ in target space. Thus, in the IR limit we seem to recover both the open string description with $D$ branes as well as the closed string description from a single underlying two dimensional quantum field theory, simply by varying a parameter. 


\subsection{The Stringy Description of Chern-Simons on $S^{3}$}

As reviewed in Sec. 2, the Chern-Simons theory on $S^{3}$ arises from the open string A-model in the vicinity of the conifold $T^{*} S^{3}$. It is convenient to describe the conifold explicitly as a subspace in $\mathbf{C}^{4}$, defined by the relation

$$
x_{1} x_{2}-x_{3} x_{4}=\mu
$$

The space has a Lagrangian $S^{3}$ submanifold of size $\mu$. (The $S^{3}$ can be seen quite explicitly after a change of variables to $\sum_{i=1}^{4} z_{i}^{2}=\mu$. Taking $z_{i}, \mu$ to be real gives the $S^{3}$ in the conifold.) The $\mu$ parameter changes the complex structure of the Calabi-Yau $T^{*} S^{3}$. However, the A-model topological string is independent of the complex structure of the manifold. In particular, all amplitudes in the topological A-model are strictly independent of $\mu$. We will find it convenient to consider the limit where $\mu \rightarrow 0$. In this limit, the equation becomes

$$
x_{1} x_{2}-x_{3} x_{4}=0
$$

and the $S^{3}$ has shrunk to a single point $x_{i}=0$ for all $i$. Therefore, in this limit the topological amplitudes for the A-model will come from open worldsheets with Dirichlet conditions $x_{i}=0$ on the boundary.

\subsection{The Linear Sigma Model Description of a Blown-up Conifold}

There exists a linear sigma model - an $N=2$ supersymmetric $U(1)$ gauge theory - whose low energy dynamics description reduces to the usual non-linear sigma model on the $S^{2}$ blown up version of the conifold [35] . One considers an $N=2 U(1)$ gauge theory with four charged chiral fields, $a_{i}, b_{i}(i=1,2)$ and charges $+1,-1$ for $a, b$ respectively. In addition the action has a FI D-term with strength $r$, as well as a $\theta$ term $i \theta \int F$ for the $U(1)$ gauge field. As far as the topological theory is concerned, all quantities will appear in the complex combination $t=r+i \theta$. Thus having $\theta \neq 0$ is equivalently to having $r \neq 0$. Then, it was shown in [35] that this model describes, in the IR limit, the blown 
up conifold, where one has an $S^{2}$ with kahler parameter $t$. The basic idea is that there is a D-term potential of the form

$$
V_{D}=e^{2}\left(\left|a_{1}\right|^{2}+\left|a_{2}\right|^{2}-\left|b_{1}\right|^{2}-\left|b_{2}\right|^{2}-r\right)^{2}
$$

Let us consider $r \neq 0$ (say $r>0$ ), then the potential forces vacuum configurations to have either $a_{1}$ or $a_{2}$ to be non-zero. In other words, we are in the Higgs phase. $a_{1} / a_{2}$ can be viewed as the complex coordinate of the $S^{2}$, and the two complex normal directions to the $S^{2}$ can be identified with the gauge invariant combinations $b_{1} a_{2}$ and $b_{2} a_{2}$. Thus the target geometry of the blown up conifold is the same as the Higgs phase of this gauge theory. (A similar story holds for $r<0$, with the $a$ 's and $b$ 's interchanged.) To see the complex structure of the conifold, note that if we consider the gauge invariant fields

$$
x_{1}=a_{1} b_{1}, \quad x_{2}=a_{2} b_{2}, \quad x_{3}=a_{1} b_{2}, \quad x_{4}=a_{2} b_{1}
$$

the Higgs branch is identified with the gauge invariant observables, modulo the relation

$$
x_{1} x_{2}-x_{3} x_{4}=0
$$

which is the defining equation of the conifold. The FI D-term corresponds to the same complex manifold given by the above equation but with a blown up $S^{2}$.

The $N=2$ gauge theory has in addition, a complex neutral scalar field $\sigma$ in the $U(1)$ gauge multiplet. In the Higgs branch this $\sigma$ field is massive, due to interactions of the form

$$
V_{i n t}=e^{2}|\sigma|^{2}\left(\left|a_{1}\right|^{2}+\left|a_{2}\right|^{2}+\left|b_{1}\right|^{2}+\left|b_{2}\right|^{2}\right)
$$

When $r \neq 0$, the Coulomb branch, corresponding to $\langle\sigma\rangle \neq 0$ is absent, because the FI term necessarily Higgses the $U(1)$ and gives masses to the $\sigma$ field due to the above interactions. Thus the $\sigma$ field is irrelevant in the IR limit.

Now, let us consider the limit where $t \rightarrow 0$. In this limit, the low energy configurations allow both the Higgs and the Coulomb branch to coexist. In the Higgs branch we have the description of the conifold as the moduli of vacuum configurations, but now with no $S^{2}$ blown up. In other words it is at the point of the conifold transition. Just as before, 
the $\sigma$ field is massive in the Higgs branch. However, we now also have the Coulomb branch, in which case $\langle\sigma\rangle \neq 0$ and where all the charged fields $a_{i}, b_{i}$ are massive and frozen in the infrared limit at $a_{i}=b_{i}=0$. The Coulomb branch is in fact a free $N=2$ abelian $U(1)$ gauge theory and therefore a trivial free theory in the infrared.

\subsection{The Topological Sigma Model and the Emer- gence of D-branes}

The issue now is to dynamically see the emergence of worldsheets with holes (and Dirichlet boundary conditions) as $t \rightarrow 0$. As we have seen from our discussion of the linear sigma model describing the conifold, it is exactly in this limit that the system will have two branches: the Higgs branch and the Coulomb branch. In $d>2$ one simply fixes the vevs of fields so that one is on one branch or the other. As is well known, the situation in $d=2$ is different, and we have to allow all possible fluctuations. In fact, in our case, as $t \rightarrow 0$, where we have both a Higgs $(\mathrm{H})$ and a Coulomb $(\mathrm{C})$ branch, we can write down spatial configurations which at $x=-\infty$ start on one branch and at $x=\infty$ end on the other. Moreover, one can arrange the energy of this configuration to be as small as one pleases. ${ }^{11}$. The basic point is that if we are in one branch, we can change vevs slowly enough over large regions so as to cost little energy. In this way, both phases coexist in the worldsheet fluctuations even in the IR. Note, in particular that the region for each phase could be as big as one wishes it to be. Thus at $t=0$ we have to consider worldsheets consisting of large regions of different phases $(\mathrm{H})$ and $(\mathrm{C})$. Approaching $t=0$ from positive values, we would have started by only having the $(\mathrm{H})$ branch, and as $t$ become smaller we can get arbitrary fluctuations involving patches in the $(\mathrm{C})$ branch. As we noted earlier, all the (charged) fields $a_{i}, b_{i}$ pick up a mass in the (C) branch and are irrelevant in the IR. In fact, their values are frozen to zero. Actually, the $(\mathrm{C})$ branch in the IR is a free abelian theory which can be integrated out leaving us with an effective theory in the $(\mathrm{H})$ branch with gaping holes corresponding to the $(\mathrm{C})$ patches. Moreover, the fact that the charged fields vanish in the $(\mathrm{C})$ branch gives Dirichlet boundary condition to the fields $a_{i}=0$ and $b_{i}=0$ at the boundary. In other words, we have the same D-brane boundary conditions that we

\footnotetext{
${ }^{11}$ We would like to thank Sidney Coleman for a discussion on this point.
} 
would have expected on the Chern-Simons side in the limit when the $S^{3} \rightarrow 0$. We are thus seeing the D-branes anticipated from our duality emerging from the worldsheet description.

For this picture to be the right one, we also need to show how the open string factor of $\left(g_{s} N\right)^{B}$ multiplying worldsheets with $B$ boundaries arises on the closed string theory side, in the limit where $g_{s} N \rightarrow 0$. Note that we are identifying $B$ with the number of $(\mathrm{C})$ patches of the linear sigma model. We will offer a speculative explanation of how this might arise. It will be important to verify this idea in a more detailed study.

Recall the identification, $g_{s} N=\lambda=\operatorname{Im} t=\theta$ in the $U(1)$ gauge theory $\left(\theta\right.$ appears in the action only in the term $\left.\theta \int F\right)$. Let us assume that the $(\mathrm{C})$ patches can be in topological sectors with vortex charge of either zero or one. In other words, since we do not have multiply charged bound vortices we exclude multiple vortex field configurations in a given Coulomb patch. If we now do a path integral in this free theory, the boson and fermion determinants will cancel up to a sign. Let us assume that in the one vortex sector we get an extra minus sign from determinants, compared to the one where the vortex field is absent. Thus the contribution from each Coulomb patch will be

$$
f=1-\exp (i \theta)
$$

In the limit $\theta \rightarrow 0$, we have $f \sim \theta$ and so we get a factor of $f^{B}=\theta^{B}=$ $\left(g_{s} N\right)^{B}$ from integrating out all the $B$ Coulomb patches. Note that the factor $1-\exp (i \theta)$ is the quantum deformation of $\theta$, in the sense of quantum groups where

$$
q-q^{-1} \rightarrow i \theta
$$

with $q=\exp (i \theta / 2)$. Note also that the contribution $1-\exp (i \theta)$ is suggestive of the fact, discussed in Sec.3 that a simple Wilson loop is expected to give $1-q^{2}$ (up to an overall factor of $q^{\alpha}$ ).

\section{Conclusions and Generalizations}

We have seen encouraging signs that the large N/Gravity correspondence involving D-branes might actually be provable at the level of 
string perturbation theory, i.e. in terms of certain facts about $2 d$ QFT's. There are clearly aspects of our argument in our particular example which need to be further developed. In particular, we obtained Dirichlet conditions from the closed string side in the $S^{3} \rightarrow 0$ limit. But one would like to check that the deformation away from this degenerate limit does not destroy the correspondence. Also it is important to verify our picture of how $g_{s} N$ factors arise from each hole.

We have been fortuitous to get an explicit closed string description of a large $N$ gauge theory which admits completely independent checks on both sides. We expect that even this case, simple as it may be, will give us more insights into the general nature of the gauge theory/geometry correspondence. It is heartening that supersymmetry did not play any crucial role in our considerations. There are also other aspects of this Chern-Simons/topological closed string correspondence that deserve study such as the detailed understanding of Wilson Loops. This might also be a mathematically fruitful connection to make. Also it would be interesting to elucidate the role of the finite size $S^{2}$ in the gauge theory.

Another natural question to consider is whether the closed topological string on a compact Calabi-Yau can be dual to a gauge theory. If there is such a description, given the IR/UV relation, and given that the large distance is naturally cutoff in the compact Calabi-Yau, one would expect the gauge theory to have a natural short distance cutoff. It would be interesting to explore such a possibility in connection with integrable lattice models and their relation to Chern-Simons theory.

There are various immediate generalizations of what we considered in this paper. For example, we can consider Chern-Simons theory on Lens spaces (or their generalizations corresponding to quotients of $S^{3}$ by discrete subgroups of $S U(2)_{L} \times S U(2)_{R}$ acting on $S^{3}$ ) and develop closed string duals (some aspects of these theories were discussed in [13]). One could also consider large $N S O$ and $S p$ gauge groups in a similarly explicit manner. The topological B-model in this closed string geometry is also worthy of study. (For example, by wrapping $D_{2}$ branes on the $S^{2}$ and considering closed strings on the $T^{*} S^{3}$ geometry, we would be studying the mirror of our present example.) Another interesting and plausibly tractable case is that with the $N=4$ topological string [4] which should be related to $2 \mathrm{~d}$ field theories (and in 
particular to Large $N$ principal chiral models which have an interesting and exactly solvable mass spectrum etc.) The closed string dual in this case describes self-dual gravity theories in $4 \mathrm{~d}$ which is also the $N=2$ string theory [25]. This raises the fascinating possibility of understanding the $(2,2)$ signature self-dual gravity theory in the large $N$ limit of a $(1,1)$ signature QFT. It might also be of interest to re-examine the string description of $Q C D_{2}$ [9] and perhaps make it as explicit as the gauge theory side (See the recent paper of Horava in [9] ).

Finally, we believe the linear sigma model approach might help in deriving the AdS/CFT correspondence. Namely, by considering a linear sigma model for an AdS background with a RR flux turned on, we expect to automatically end up with the D-brane rules of open string theory in the limit where $g_{s} N \rightarrow 0$, presumably via a two phase system. This would then be a proof of the AdS/CFT correspondence using perturbative string theory techniques. The main task is to first find a convenient linear sigma model description of the AdS background with a RR flux. Then it is a matter of dynamics to determine whether or not the behaviour as $g_{s} N \rightarrow 0$ allows us to, say, integrate a trivial phase out, leaving us effectively with a worldsheet with Dirichlet boundaries. Moreover, the fact that we need to take $\alpha^{\prime} \rightarrow 0$ is somewhat similar to having a topological limit on the gauge theory side.

We would like to thank M. Bershadsky, S. Coleman, S. Katz, A. Lawrence, J. Maldacena, T. Pantev, A. Strominger, C. Taubes and S.T. Yau for valuable discussions.

The research of R.G. is supported by DOE grant DE-FG02-91 ER40654 and that of C.V. is supported in part by NSF grant PHY98-02709.

\section{References}

[1] A general reference on knot theory is Knots and Physics, by L.Kauffman, World Scientific, (1993).

[2] P. Aspinwall, B. Greene, and D. Morrison, Calabi-Yau Moduli Space, Mirror Manifolds and Spacetime Topology Change in String Theory, Nucl. Phys., B416 (1994), 414. 
[3] S. Axelrod and I. Singer, Chern-Simons Perturbation Theory II, J. Diff. Geom., 39 (1994), 173.

[4] N. Berkovits and C. Vafa, N=4 Topological Strings, Nucl. Phys., B433 (1995), 123.

[5] M.Bershadsky, S.Cecotti, H.Ooguri, C.Vafa , Holomorphic Anomalies in Topological Field Theories, Nucl. Phys., B405 (1993), 279.

[6] M. Bershadsky, S. Cecotti,H. Ooguri, C. Vafa , Kodaira-Spencer Theory of Gravity and Exact Results for Quantum String Amplitudes, Comm. Math. Phys., 165 (1994), 311.

[7] M. Bershadsky and V. Sadov, Theory of Kähler Gravity, Int. J. Mod. Phys., A11 (1996), 4689.

[8] P. Candelas, X.C. de la Ossa, P.S. Green, and L. Parkes, A Pair of Calabi-Yau Manifolds as an Exactly Solube Superconformal Theory, Nucl. Phys., B 359 (1991), 21.

[9] S. Cordes, G. Moore, S. Ramgoolam, Large N $2 D$ Yang-Mills Theory and Topological String Theory, Commun. Math. Phys., 185 (1997), 543;

P. Horava, Topological Rigid String Theory and Two Dimensional QCD, Nucl. Phys., B463 (1996), 238; On QCD String Theory and AdS Dynamics, hep-th/9811028.

[10] M.R. Douglas, Chern-Simons-Witten Theory as a Topological Fermi Liquid, hep-th/9403119.

[11] M. Douglas, V. Kazakov, Large N Phase Transition in Continuum $Q C D_{2}$, Phys. Lett., B319 (1993), 219.

[12] C. Faber and R. Pandharipande, Hodge integrals and GromovWitten theory, math.AG/9810173.

[13] R. Gopakumar, C. Vafa, Branes and Fundamental Groups, hep-th/9712048.

[14] R. Gopakumar, C. Vafa, M-Theory and Topological Strings-I, hep-th/9809187. 
[15] M. Green and J. Polchinski, Summing Over World-Sheet Boundaries, Phys. Lett., B335 (1994), 377.

[16] B. Greene, C. Vafa, and N. Warner, Calabi-Yau Manifolds and Renormalization Group Flows, Nucl. Phys., B324 (1989), 371.

[17] D. Gross, Nucl. Phys., B400 (1993), 161;

D. Gross, W. Taylor, Nucl. Phys.m B400 (1993), 181; B403 (1993), 395.

[18] S.S. Gubser, I.R. Klebanov, and A.M. Polyakov, Gauge Theory Correlators from Non-Critical String Theory, Phys. Lett., B428 (1998), 105.

[19] S. Hosono et. al., Mirror Symmetry, Mirror Map and Applications to Complete Intersection Calabi-Yau Spaces, Mirror Symmetry II, ed. by B. Greene and S.-T. Yau, AMS/IP.

[20] S. Katz, private communication.

[21] J. Maldacena, The Large N Limit of Superconformal Field Theories and Supergravity, Adv. Theor. Math. Phys., 2 (1998), 231.

[22] J. Maldacena, Wilson loops in large $N$ field theories, Phys. Rev. Lett., 80 (1998), 4859;

S-J. Rey, J.Yee, Macroscopic Strings as Heavy Quarks of Large $N$ Gauge Theory and Anti-de Sitter Supergravity, hep-th/9803001.

[23] M. Marino and G. Moore, Counting higher genus curves in a Calabi-Yau manifold, hep-th/9808131.

[24] E. Martinec, Criticality, Catastrophes, and Compactifications, in Physics and Mathematics of Strings, ed. L. Brink, D. Friedan and A.M. Polyakov (World Scientific, 1990).

[25] H. Ooguri and C. Vafa, Geometry of N=2 Strings, Nucl. Phys., B361 (1991), 469.

[26] V. Periwal, Topological Closed-string Interpretation of ChernSimons Theory, Phys. Rev. Lett., 71 (1993), 1295.

[27] K.Skenderis, M. Henningsson, The Holographic Weyl anomaly, hep-th/9806087. 
[28] L. Susskind, E. Witten, The Holographic Bound in Anti-de Sitter Space, hep-th/9805114.

[29] C. Taubes, private communication.

[30] G. 't Hooft, A Planar Diagram Theory for Strong Interactions, Nucl. Phys., 72 (1974), 461.

[31] G. 't Hooft, On the Convergence of Planar Diagram Expansions, Commun. Math. Phys., 86 (1982), 449.

[32] E. Witten, Topological Sigma Model, Comm. Math. Phys., 118 (1988), 411.

[33] E. Witten, Chern-Simons Gauge Theory As A String Theory, hep-th/9207094.

[34] E. Witten, Quantum Field theory and the Jones Polynomial, Commun. Math. Phys., 121 (1989), 351.

[35] E. Witten, Phases of $N=2$ Theories in 2 Dimensions, Nucl. Phys., B403 (1993), 159.

[36] E. Witten, Anti De Sitter Space And Holography, Adv. Theor. Math. Phys., 2 (1998), 253. 